mansasa

glyndŵn

Glyndŵr University

Glyndŵr University Research Online

Computing

Computer Science

$1-1-2002$

\title{
Choosing threshold levels for electromagnetic hazards
}

Peter S. Excell

Glyndwr University, p.excell@glyndwr.ac.uk

Follow this and additional works at: http://epubs.glyndwr.ac.uk/cair

Part of the Computational Engineering Commons, and the Computer Sciences Commons

\section{Recommended Citation}

Excell, P. S. (2002)'Choosing threshold levels for electromagnetic hazards'. IEEE Technology and Society Magazine, 21(4), 32-39.

This Article is brought to you for free and open access by the Computer Science at Glyndŵr University Research Online. It has been accepted for inclusion in Computing by an authorized administrator of Glyndŵr University Research Online. For more information, please contact

d.jepson@glyndwr.ac.uk. 


\title{
Choosing threshold levels for electromagnetic hazards
}

\begin{abstract}
The concept of electromagnetic hazards is today frequently taken to refer to direct effects on biological tissue. However, several other effects are possible and have been the subject of a substantial amount of research in earlier decades. One of these is the hazard of ignition of flammable gas/vapor mixtures due to sparks resulting from radiofrequency currents flowing in metallic structures. Although the physics of the mechanism linking power from a radio transmitter to ignition of a flammable mixture is relatively straightforward, compared with bioelectromagnetic effects, there are many uncertainties and poorly-understood steps in the process, leading to considerable difficulties for committees tasked with setting safety limits. The author has experience of such committees, notably that concerned with British Standard 6656, and the way in which these difficulties were addressed may give useful insights for the regulation of other hazards.
\end{abstract}

\section{Keywords}

electromagnetic hazards, radiofrequency currents, flammable gas/vapor mixtures, British Standard 6656, safety limits

\section{Disciplines \\ Computational Engineering | Computer Sciences}

\section{Comments}

(C2002 IEEE. Personal use of this material is permitted. However, permission to reprint/republish this material for advertising or promotional purposes or for creating new collective works for resale or redistribution to servers or lists, or to reuse any copyrighted component of this work in other works must be obtained from the IEEE. This material is presented to ensure timely dissemination of scholarly and technical work. Copyright and all rights therein are retained by authors or by other copyright holders. All persons copying this information are expected to adhere to the terms and constraints invoked by each author's copyright. In most cases, these works may not be reposted without the explicit permission of the copyright holder. The paper was published by IEEE Technology and Society Magazine in 2002 and was sponsored by IEEE Society on Social Implications of Technology. The definitive version is available at http://ieeexplore.ieee.org 

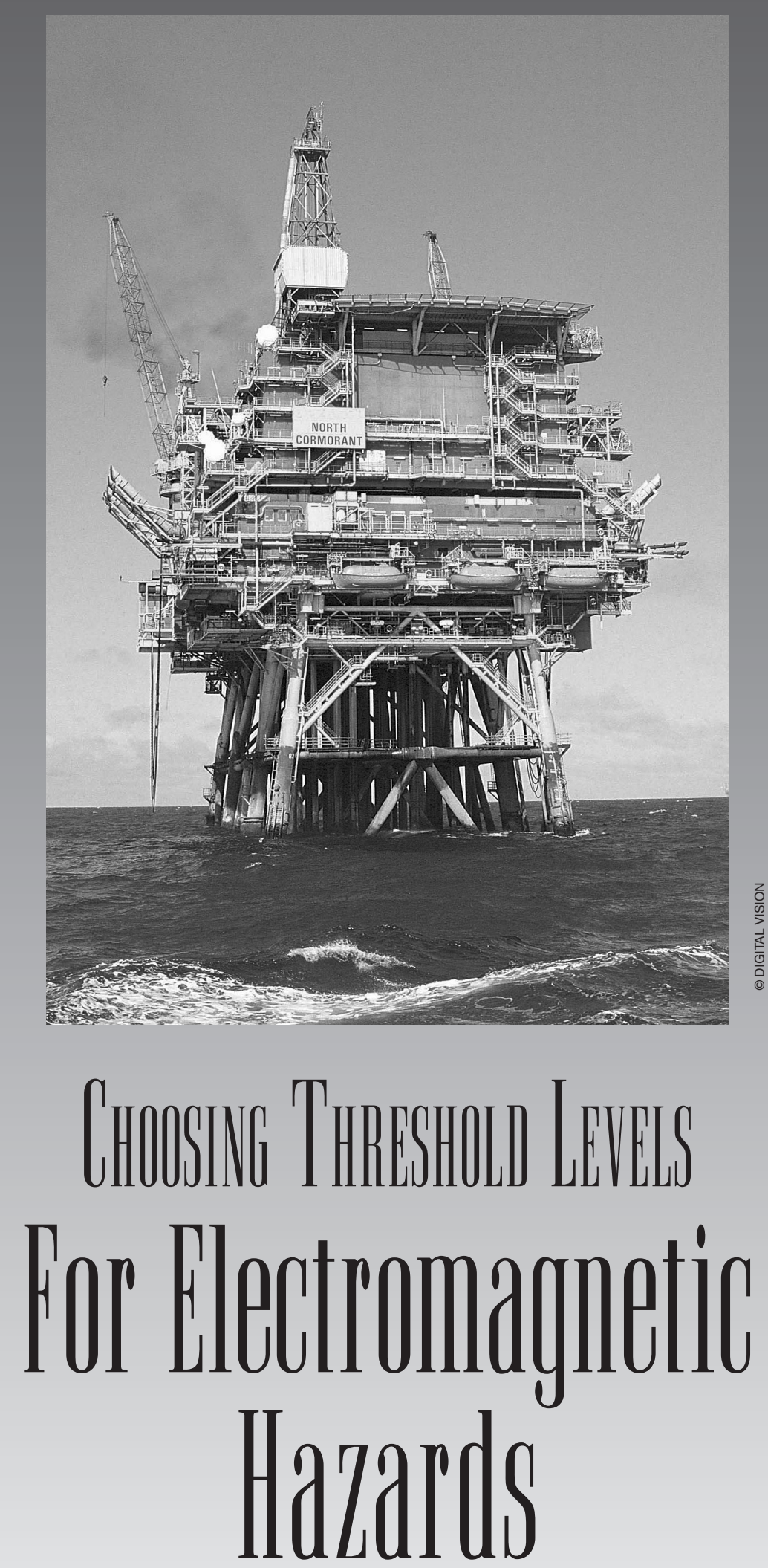

Peter S. Excell 
T he concept of "electromagnetic hazards" is today frequently taken to refer to direct effects on biological tissue. However, several other effects are possible and have been the subject of a substantial amount of research in earlier decades. One of these is the hazard of ignition of flammable gas/vapor mixtures due to sparks resulting from radio frequency currents flowing in metallic structures. Although the physics of the mechanism linking power from a radio transmitter to ignition of a flammable mixture is relatively straightforward, compared with bioelectromagnetic effects, there are many uncertainties and poorly-understood steps in the process, leading to considerable difficulties for committees tasked with setting safety limits. The author has experience of such committees, notably that concerned with British Standard 6656 [1], and the way in which these difficulties were addressed may give useful insights for the regulation of other hazards.

\section{MeChaniSM OF THE HAZARD}

Radio transmitters radiate power into their environment in the form of electromagnetic fields. In the intended mode of functioning, these fields interact with metallic receiving antennas, generating currents in the desired band of frequencies. These currents, which are extremely weak in typical cases, can then be amplified and demodulated in suitable electronic circuits such that the desired audio, video or data information is recreated in baseband form.

The electromagnetic fields cannot distinguish between a receiving antenna and any other metallic structure, and hence radio frequen-

The author is with the University of Bradford, Department of Electronic Imaging and Media Communications Bradford, UK BD7 1 DP; email:P.S.Excell@Bradford.ac.uk cy currents are induced in all metallic structures encountered in the propagation of the radiated waves. Relatively close to a transmitter, these currents can be relatively strong and they can lead to substantial voltages at terminating ends and discontinuities in the metallic structures. Clearly, in extreme cases, a sufficiently high voltage may be generated such that a spark could jump a gap between two metallic structures. In practice, this requires such high power that it is only viable extremely close to powerful transmitters. A much more significant mechanism for the production sparks is the so-called "break spark," in which some form of opening contact occurs within the metallic structure or between two separate structures that are initially in contact. This mechanism allows the spark to be started with a relatively small voltage, creating an initial plasma which can then be expanded as the metallic parts are drawn apart.

If the spark is surrounded adventitiously by a flammable gas/air mixture there is the possibility that the mixture may be ignited and cause an explosion. This is obviously a matter of serious concern, although it will only occur if the gas/air ratio is within a known range of values and the power generated in the spark is above a known threshold. Although very few incidents of explosions caused by his mechanism have ever been recorded, it is physically obvious that the mechanism is entirely possible and hence it became a source of major concern to military authorities, and later to the oil and gas industries. Military concerns were predominantly focused on the potential for ignition during the refueling of aircraft, especially on the deck of an aircraft carrier, where there are many relatively high power radio transmitters close to an aircraft on the deck. Concern in the oil and gas industries was greatly boosted when offshore fields began to be developed. Offshore exploration and production platforms have to carry a wide range of radio transmitters radiating moderately high powers, but they are located very close to potential sources of flammable gas/air mixture production. It is also pertinent to note that the density of investment and personnel on an offshore platform means that the basic financial risk involved is very high, and this is augmented many times over by the potential cost of the loss of production if an accident were to occur.

The basic physical mechanism involves radiation of power from a transmitter, propagation through space, and reception by a metallic structure acting as an unintended receiving antenna (URA). This basic mechanism is summarized in the Friis transmission formula:

$$
\frac{P_{R}}{P_{T}}=\frac{G_{T} G_{R} \lambda^{2}}{(4 \pi r)^{2}}
$$

Where $P_{T}$ is the power radiated by the transmitting antenna, $P_{R}$ is the power available to a matched load at the receiving antenna (URA), $G_{T}$ is the gain of the transmitting antenna, $G_{R}$ is the gain of the URA, 1 is the wavelength and $r$ is the distance between the transmitting antenna and the URA.

Of these variables, most are well established, but $G_{R}$ and the limiting safe value of $P_{R}$ are problematic. After a lengthy period of debate and experimentation, it was found that limiting maximum safe values of power dissipated in a spark could be specified for a range of the most common flammable mixtures. These mixtures have to be assumed to be in admixture with normal air (pure oxygen, for instance, greatly reduces the threshold for ignition) and three broad classes of common flammables are defined, as had previously been found in experiments to find limiting values of pulsed ignition energy from transient (e.g., electrostatic) sparks. The flammable substance are divided into 'Groups', having broadly similar minimum ignition energies (MIEs): 
Olishorore explonation and production

platiforms have to carring a wide ranthe of

radio transmilteres radiating moderatelly

hingh powers, but thet are located ver"y

close to potential sourcess of flammadide

gas/air mixiluere productition.

Group I is a special category for natural gas released in coal mines ('firedamp'); Group IIA covers most common gaseous or vaporizable flammables, especially the saturated hydrocarbons (alkanes); Group IIB gases have lower MIEs, the only common example being ethylene (ethene; but note that it is by no means the case that all alkenes fall into this group); Group IIC covers substances with the lowest MIEs, which are hydrogen, acetylene, carbon disulphide and a few other exotic substances [1].

The physics determining the spark ignition power threshold is complex. Ignition requires a selfsustaining flame front to be developed in the flammable mixture and, if the incipient ignition is regarded as a spherical fireball, it can be visualized that a spark with an energy below the ignition threshold (MIE) will cause some combustion in its spark channel but that the fireball will be so small that, as it expands, it cools more rapidly than heat is gained by the exothermic combustion reaction. Thus, a sufficient initial burst of electrical heat is required to augment the combustion reaction such that the fireball remains hot enough to cause continuing combustion at its outer surface until it has grown sufficiently large to be self-sustaining. Studies of this mechanism were conducted extensively with pulsed (dc) sparks and these elucidated the behavior of the different flammable mixture groups, although the mechanism, and the reasons for the differing MIEs, are still not fully understood. Relating this work to sparks caused by continuously available radio frequency power caused considerable difficulties. It was realised at an early stage that a spark between a fixed electrode gap would require an improbably high voltage unless the electrodes were extremely close together, in which case they would cause quenching of the expanding flame front and hence suppression of the self-sustaining reaction. It had been discovered in experiments on explosion safety of dc and power frequency equipment that the most severe condition was the so-called break spark, in which conductors initially in contact were pulled apart, at least one of them with a sprung "flicking" motion such that a spark could be struck with a very low source voltage at the instant of breaking, but then the flicking of the electrode removed its quenching action from the flame front [2].

The extensive work at dc and power frequencies had not, however, been quantified in terms of power, as was the most logical parameter for radio frequency situations. It was concluded that new experiments with radio frequency sources were needed, and several groups modified the existing break spark test equipment to reduce its capacitive loading effect on radio frequency sources: experiments were than undertaken over a range of frequencies to determine whether these had an influence on the power required for ignition. Early experiments were undertaken with $50-\mathrm{W}$ source impedances, as seemed a logical choice, but the results showed somewhat surprisingly high powers to be required and an unexplained frequency dependence [3], [4].

A landmark experiment at the British Navy's Admiralty Surface Weapons Establishment showed that ignition could be caused with far lower powers if the source impedance were made much higher than $50 \mathrm{~W}$, such that the voltage available for a given power was greatly augmented. The higher source voltage enabled the spark to continue burning for a longer period as the electrodes separated, thus allowing more energy to be dumped into the transient spark and also enabling the quenching effect of the electrodes to be removed. Following this, some new series of quantitative experiments with high source impedances were undertaken and it was found that the power required for ignition was much lower than with $50-\mathrm{W}$ sources and was now essentially independent of frequency [5]. A set of powers for the three flammability classes was determined by experiment (Table I) and these are now universally accepted, although the physical connection between these powers and the energies required for ignition is still very complex and not fully understood, especially since the match condition between the spark and the radio frequency source is changing continuously during the spark process.

It may be noted that, for pulsed radars, an energy based criterion can still be used, since the spark can safely be assumed to be caused by a single pulse. Nonetheless, the use of the energies determined in dc experiments leads to difficulties (see below) and hence higher values are used in current safety standards.

The issue of the gain of the URA poses still greater difficulties. In initial work, the groups developing 
safety standards presumed that a gain of around 1.5 was reasonable, since this is the value for an electrically small dipole or loop antenna, and the value for a half wavelength dipole antenna is not greatly different (1.64). However, use of this value leads to the prediction of the potential for explosions at improbably large distances from the transmitter at low frequencies. For example, if the case of a typical AM radio transmitter is considered (ignoring ground losses, which have a significant but not overwhelming effect) the Friis transmission formula may be rearranged to give a minimum safe distance from the transmitter for establishments handling flammable mixtures:

$$
r=\frac{\lambda}{4 \pi} \sqrt{\frac{P_{T} G_{T} G_{R}}{P_{R}}}
$$

Taking realistic values of $P_{T}=$ $200 \mathrm{~kW}, G_{T}=5$ (e.g., for an antifading monopole antenna), $1=300$ m (i.e., $1 \mathrm{MHz}$ ) and $P_{R}=8 \mathrm{~W}$ (the value found for the most common flammable substances), then (2) predicts a minimum safe distance from the transmitter of $10.3 \mathrm{~km}$ (larger if modulation is taken into account). This is plainly ridiculous, since it would suggest that the large number of (for example) gasoline filling stations that have survived for many years around such transmitters at closer distances should have been the scenes of many tragic explosions.

In fact, only two fires or explosions are known to have occurred which have definitely been ascribed to radio frequency ignition [6], although several more instances of potentially hazardous sparking have been observed in situations where there was fortunately no flammable mixture. The fact that hardly any such explosions have occurred implies a need to reexamine the physics. Certainly propagation losses can be added to the model and they give some reduction in the predicted power appearing in the URA, but this effect is modest at the relatively short distances concerned. It was then noticed that receiving antenna efficiency had necessarily to be taken into account since use of the simplistic gain value of the order of 1.5 would lead to quite ridiculous predictions at very low frequencies. The fundamental difference in efficiency between electrically small and electrically large antennas thus had to be invoked, electrically small URAs being dominated by their low efficiency, causing a much reduced value of gain. In quantifying this, the concept of the "reasonable worst case (RWC) assumption" becomes important, although it was a guiding principle in all considerations of safety standards for this hazard mechanism.

Clearly, in improbably exotic cases, one might postulate the existence of (for example) huge parabolic reflectors, supergain antennas, or highly efficient antennas using superconductors. Certainly the latter two of these can be dismissed as being completely ridiculous in a URA. The possibility of an adventitiously occurring reflector is not quite impossible, but "engineering judgment" concluded that it was so improbable that it could be ruled out. This decision, however, illustrates the introduction of a subjective element in decisions on safety thresholds.

Further such decisions had to be taken in deriving formulas for the RWC efficiency of electrically small URAs. The loop antenna was first chosen as the canonical example, since it can be constituted by a continuous metallic structure (e.g., a crane on a metallic deck), whereas a dipole-like structure requires an elevated insulated metallic conductor. The probable value for loss resistance in the loop antenna then had to be derived, by assuming that it was made of steel, and hence had substantially more loss than copper, and choosing a range of representative values for its effective cross- sectional diameter. The inclusion of the efficiency completely changed the frequency characteristic of the Friis formula: if efficiency is not considered, this gives a received power that is proportional to $f^{-2}$. However, a detailed analysis of the efficiency of the loop antenna shows that it is proportional to $f^{3.5}$, thus giving an overall dependence on $f^{1.5}$ [7]. The rather surprising $f^{3.5}$ dependence follows from the facts that the radiation resistance of a loop antenna is proportional to $f^{4}$, but the ohmic resistance of the conductors, as modified by the skin effect, is proportional to $\div f$, and the efficiency is proportional to their ratio when the loss is dominant.

This modification eliminates the problem of improbably high received powers at low frequencies, but it introduces the issue of cost/benefit trade-offs in deciding what is a realistic hazard. Clearly, no one could realistically argue that the antenna might be made of superconductor or silver, but the possibility of its construction from copper or aluminium rather than steel obviously has a nonzero probability. It was found, however, that the use of the parameters for steel gave output powers from a URA that were still about three times larger than were found in experimental measurements and hence it was concluded that the use of the steel formula contained sufficient safety factors to cover for rare cases of structures with higher conductivity.

Another variable factor that was considered was the polarization match of the loop antenna to the incident radiation. A vertical loop such as a crane is polarizationmatched to incident vertically-polarized radiation when the plane of the loop is normal to the direction of the (horizontal) magnetic field vector. Clearly, the probability of achieving perfect polarization match is very small, but given the shape of the field pattern of such an antenna, the polarization matching factor (for power) will be between 0.5 and 1.0 for $50 \%$ 
of possible orientation angles, and hence a worst-case assumption that it is always 1.0 is not unreasonable.

The electrically small loop antenna is inherently inductive and needs to be resonated with a tuning capacitance if it is to act as a reasonably effective antenna. Here, much more engineering judgement had to be applied. Taking the crane as the canonical RWC example, it was reasoned that it could be lifting a metallic load from a metallic surface (e.g., the deck of an offshore platform), and hence a parasitic capacitance could be formed that would cause the antenna to pass through the tuned condition: a trailing wire from the load could then cause a break spark event. An alternative line of argument might have been to say that the possibility of such tuning was too improbable and that the antenna should always be regarded as untuned. This view was not taken by the relevant committee, but this illustrates the arbitrariness of many decisions based on engineering judgement.

For electrically large antennas the possibility of near-unity efficiency is very high, since the radiation resistance is relatively large. Unity efficiency was thus assumed, but a different problem arises in the choice of the value of gain. A decision on this was reached on the basis of judgement following a study of electrically long dipole antennas [8]. Studying the envelope of the graphs of maximum directivity as a wire dipole antenna was extended in length, it was noted that the gradient of this envelope declines somewhat for longer lengths such that it was considered that a value of 10 for the maximum gain was a reasonable choice, bearing in mind that the width of the (axisymmetric) main lobe would be relatively small, making orientation critical, and hence the probability of optimum orientation small. Note, however, that this reasoning is based on qualitative assessments of probabilities.
Outside of electrical considerations, a major effect contributing to a low rate of explosions is the simple fact that flammable atmospheres are not normally present, and only occur when some fault situation has developed. This situation has a very low probability of occurrence, but committees have generally felt obliged to take the probability as unity.

Another non-electrical consideration is the probability of occurrence of the breaking contact: again, committees have assigned this an effective value of unity, although realistic values are far smaller.

The large number of concatenated worst-case judgements taken by consensus of the committees is not very satisfactory from a philosophical standpoint. The judgements were made on a subjective and qualitative basis, and the possibility of error in them would be difficult to rule out. Due to the fact that a limited range of measurements showed that measured received powers were still substantially lower than those found from theoretical predictions, no safety factors were included in the treatment, despite this normally being the case in safety standards.

A particular problem occurred with the power received from pulsed radars. Ideally the energy in the pulse at the safe limit should be similar to the energy in well-established dc spark ignition studies, and this had been shown to be the case in laboratory conditions [9]. However, using this value led to implausibly large diameters of hazard zones around radar antennas. To overcome this, a series of experiments were undertaken which showed that far less induced power was found and hence the level of ignition power deemed to be hazardous was somewhat arbitrarily increased to a substantially higher value in order to reduce the diameter of the 'at risk' zone [10]. The rationale for this was that the break spark mechanism would absorb some of the energy and quench part of the flame front, but the experiments on which this was based were far from comprehensive.

Particular complexities arise where multiple overlaid transmissions at different frequencies are considered. This then begs the question whether they should be added in voltage or power terms and whether the antenna should be considered to be tuned to one of them and detuned with respect to others. Power addition and a limited attenuation of untuned frequencies were adopted in [1], using a complex formula based on further RWC treatments.

Matters become even more complicated when mobile transmitters are considered. This was vividly illustrated in assessments of possible hazards on offshore drilling platforms. Such platforms may have many tens of VHF or UHF walkie-talkies, typically radiating of the order of $1 \mathrm{~W}$. The hypothetical question must then be asked "supposing that 20 or more of these walkie-talkies were brought together and keyed simultaneously, could they induce enough power in a URA to cause an ignition?" This raises further questions, such as the possibility of them all operating on the same frequency simultaneously and the effect of the mutual coupling on mismatch at the power amplifiers. It appears virtually impossible for them to add coherently, but incoherent (power based) addition at closely spaced frequencies looks entirely possible. However, it is very clear that a balance has to be struck between the tiny risk of multiple adjacent operation of these transmitters against the importance of having such transmitters available widely dispersed around the platform, since the possibility of some more normal accident has a much higher probability and the walkie-talkies are the ideal means of reporting such an accident so that an immediate response can be initiated. This issue is also a matter of concern for the safety of 
electroexplosive detonators on offshore platforms [11].

Modulation of the waveform does not cause great problems. The dc experiments have shown that there is a "critical time" within which the energy must be delivered if it is to have maximum effect. If it is delivered more slowly, then additional energy is required to cause ignition, due to thermal loss from the fireball. Typical critical times are: $200 \mathrm{~ms}$ for Group IIA, $100 \mathrm{~ms}$ for Group IIB, and $20 \mathrm{~ms}$ for Group IIC. The RWC view means that amplitude modulated waveforms that have a lowest-order periodicity that is equal to or greater than these times must be assessed at the modulation peak. For others, such as FM, the mean power can be used. Pulsed radars are exceptions, and they can be handled on a pulse energy basis, as discussed above.

The issue of possible intensification of the incident field by reflection and focusing has been briefly mentioned above in the context of improbable parabolic reflectors. However, other reflection-intensification scenarios are possible which are less improbable. For instance, reflection by a single metallic sheet will cause a standing wave that will give a 6-dB intensification of the electric field strength, but does this equate to an amplification of the available power by four times, or will the output impedance of the URA be modified by coupling to its image in reflecting surface? Almost equally likely is the possibility of occurrence of a "corner reflector" structure, formed by a $90 \infty$ concave angle between conducting plates. This is a well-known structure in antenna design, giving a gain of about $12 \mathrm{dBi}$ [12]. This is a simple structure which will occur quite commonly in the real world and which cannot be dismissed as improbable, unlike the parabolic reflector. A worse-case view might argue that this structure is realistically quite likely to occur and hence that the possibility of a ten times power amplification should be allowed for (i.e., $12 \mathrm{dBi}$ minus the gain of the basic loop antenna, which is approximately $2 \mathrm{dBi}$ ). However, in practice this again leads to unrealistically large zones of risk and, since little evidence of the effect was found in practice, the committee decision was not to allow for any enhancement due to reflections in theoretical treatments.

\section{DISCUSSION OF THE ETHICAL Issues}

Clearly, those charged with the responsibility for drafting safety standards to protect against the possibility of occurrence of hazards of this nature have "people's lives in their hands." Conventionally, these matters are decided by committees, of which the present author has been a member of a number in the U.K. His task was frequently to present the physical arguments to the committee, but equally he understood the pragmatic difficulty that very few real accidents of this type have occurred in the past and that an over-emphasis on safety could cause unjustifiable restraints on communications systems which might either be an important factor in the safety of individuals or that performed a valuable service in society, and whose loss might be a detriment that society would wish to balance against acceptance of a very small level of risk.

These dilemmas are not new. In the nuclear and chemical industries, in particular, there has long been a realization that absolute safety is impossible to achieve, except by closing down the industry completely. In these cases, a very sophisticated level of analysis has been applied in an attempt to quantify the probability of occurrence of all accident mechanisms that can be envisaged [13]. Where necessary, steps are then taken to modify processes in order to reduce these probabilities below an acceptable threshold. The threshold of accept- ability is basically determined by risks that the public deems acceptable, multiplied by a safety factor. For many years, the norms for acceptable risk have been the rate of death by disease, which has been similar to the rate of death in road accidents (excluding motorcycles, which are significantly more hazardous). Figures vary, but a fatality rate of around one death per 10000 person-years is a typical figure. The philosophy of the hazardous industries is then to demonstrate that the probability of death due to their processes (the fatal accident rate, FAR) is essentially "in the noise" compared with these accepted risks, so a FAR of about 1000 times lower is considered a reasonable target, although figures vary.

Whether this is a moral approach is effectively a matter for political decision. Clearly, if the disease and/or road accident rates death rates could be significantly reduced then this would bring industrial hazards up to a higher level of significance and could put some onus on the proprietors of such businesses to spend more funds to reduce their predicted FAR. Conversely, FAR arguments can also justifiably be used to inhibit excessive spending on very small risks, using the argument that the resources could be better spent on road safety improvements, medical research, building more hospitals, etc.

Applying these concepts to electromagnetic ignition hazards, there is at present a lack of a complete probabilistic analysis of the occurrence of a hazard, although some efforts have been made to initiate the work [14]-[16]. In fact, in the language used above, many phenomena were deemed to be either so improbable as to be negligible, or alternatively reasonably probable within a worst-case scenario, but all of these decisions were made by use of professional engineering judgement by members of committees and almost no rigorous mathematical analysis of probabilities 


\begin{tabular}{|l|c|c|c|}
\hline \multicolumn{3}{|c|}{ MINIMUM IGNITION ENERGIES (MIE) AND RF IGNITION POWERS FOR COMMON FLAMMABLES } \\
\hline MIE Group & $\begin{array}{c}\text { MIE } \\
(\mu \mathbf{J})\end{array}$ & $\begin{array}{c}\text { RF ignition power } \\
\text { (W) }\end{array}$ & $\begin{array}{c}\text { Radar MIE ( } \mu \text { J), as } \\
\text { given in [1] }\end{array}$ \\
\hline $\begin{array}{l}\text { IIA (e.g. methane, propane, butane, } \\
\text { gasoline vapour, benzene, acetone) }\end{array}$ & $200-280$ & 6 & 950 \\
\hline $\begin{array}{l}\text { IIB (e.g. ethylene, acrylonitrile, } \\
\text { diethyl ether, hydrogen cyanide, coal gas) }\end{array}$ & $\sim 100$ & 3.5 & 250 \\
\hline IIC (e.g. hydrogen, acetylene, carbon disulphide) & $\sim 20$ & 2 & 50 \\
\hline
\end{tabular}

was undertaken. In fact, much the same can be said of the wide generality of electromagnetic compatibility problems, almost all of which are still assessed on a rather crude deterministic go/no-go approach using engineering judgement. Many such effects can be just as safety-critical as explosion hazards, but with a higher level of probability of occurrence, e.g., interference in vehicle anti-lock braking and engine management systems; interference to aircraft control systems; interference to electrical control systems in safety-critical chemical and nuclear plants.

Consideration of the probabilistic hazards-analysis approach implies that many electromagnetic compatibility/hazards effects should ideally be considered to be reliability problems, in which an unwanted/hazardous event corresponds to a "failure," exactly like failure of a transistor gate in a safety-critical electronic system. Inasmuch as reliability theory is quite highly developed in a probabilistic form, it may be said that electromagnetic compatibility and electromagnetic hazards analyses are significantly deficient and rather crude, and that conversion to a probabilistic basis appears to be a highly desirable development for the future.

\section{LEGAL AND \\ EnVironmental Aspects}

Electromagnetic radiation for communications is probably unique, in that it is an environmental disturbance that is broadcast out- side the perimeter of its originator's site as a necessary part of its function. It undoubtedly has the potential to cause certain types of hazard (if only in rare circumstances), and yet the victims of any possible accident do not appear to have any direct means for redress against the broadcaster or the national radio regulation authorities (certainly not in the English legal system, and it is believed that this is generally the case). This observation is offered purely as a matter of interest, not as an indication of a deficiency needing to be rectified; nonetheless, it is a point that may merit further consideration, since there seem to be significant points of difference with, for instance, electricity and water supply utilities, sound, or environmental pollutants.

\section{RELATIONSHIP TO OTHER HAZARDS}

Another form of potential electromagnetic hazard that has not yet been mentioned is that of the direct action of electromagnetic fields on human tissue. This has some similarity with the electromagnetic ignition hazard, but obviously differs in the nature of the "receiver." Much the same considerations apply to the transmitted power and the propagation path, although those parameters are essentially deterministic and uncontroversial. The issues of intensification of the incident field due to reflection and focusing are largely identical: it is noteworthy that these seem to have been rarely discussed in the bio- electromagnetics context, although some studies are known to have considered intensification of the field due to reflection from the surfaces of buildings [17].

The mechanism in the "receiver" is very different, since human tissue does not really behave in any close analogy with a receiving antenna. For the well-understood thermal hazard, there is a relatively well understood deterministic connection between the input field strength and the maximum specific absorption rate (SAR), although this certainly will have an orientation dependence which has not been investigated in great detail, due to the application of the RWC principle, in which only a realistic worst case orientation is normally considered.

For the putative non-thermal effects, the biophysics at present admits very little scope for explanation of any mechanism, although researchers continue to look for the possibility of subtle effects. It thus becomes difficult to apply either deterministic or probabilistic approaches to the setting of safety standards and this is the case where the "precautionary principle" has been invoked by several bodies, to suggest that a certain limits should be placed on SAR, "just in case" a hazard mechanism might be discovered in the future. Work to try to discover a clear-cut, generallyagreed mechanism continues, but it is unlikely but there will be any consensus on this, positive or negative, for some years yet.

Consideration of the probabilis- 
tic approaches to reliability and hazards assessment in inherently potentially dangerous industries suggests that there might be some utility in examining an approach in which it would be assumed, as temporary postulate, that some kind of non-thermal mechanism had been discovered and agreed upon but that its probability of causing a significant problem is extremely low. In the absence of knowledge about such a mechanism, would it then be possible to postulate two models (threshold-type and dose-response related) and assess the probability of occurrence of a problem which could then be compared with accepted low-probability occurrences (e.g. FAR)? This would certainly be possible in principle for human beings existing in the stochastic field environment represented by the relative position of base stations with respect to the quasirandom movements of a human being in normal daily life. However, the situation with respect to hand-held mobile telephones is more difficult, since the geometrical aspects are essentially deterministic in that case and there is only a stochastic aspect with respect to the periods of use. Nonetheless, this idea may be worthy of further exploration since the limited experimental evidence suggests that the probability of occurrence of any putative effect is likely to be extremely low and quite possibly well into the FAR "noise level."

\section{ApPlication of TeChNiQue TO BIOELECTROMAGNETIC HAZARDS}

Experience drawn from many years of research and involvement with standards-making bodies in the area of electromagnetic ignition hazards has been reviewed and an attempt made to draw generic lessons. Specific lessons include the difficulty of relating physical theory to actual experience, the wisdom or otherwise of the use of safety factors (analogous to the "precautionary principle") and the desirability, in principle, of evaluating all hazards probabilistically, so that they can be compared with generally accepted hazards. It seems likely that much work remains to be done on probabilistic assessment, especially in wider aspects of electromagnetic compatibility in safety-critical systems. Application of this technique to the issue of bioelectromagnetic hazards is problematic, due to the lack of any as-yet agreed physical mechanism for the putative nonthermal effects. However, it has been a suggested that an investigation of such a probabilistic approach, using arbitrarily postulated parameters for the characteristics of some non-thermal effect that might be discovered in the future, might have some utility or yield some insights.

The message for the precautionary principle appears to be that it should be applied with great caution. For the ignition hazard, the fact that experience of hazardous incidents has been far lower than appears to be predicted by the physical theory has been used as a reason for largely discarding safety factors, and in some cases for adopting much higher hazard threshold levels than appear to be indicated by physical theory. The principle is also well established that restriction of a beneficial technology on the basis of a weakly justified theory of a hazard mechanism may be more damaging than tolerating the hazard: this relates strongly to the philosophy in inherently hazardous industries where the achievement of total safety is fully accepted as being impossible and instead effort is directed towards maintaining the risk of any severe effects below a socially acceptable level. If any subtle bioelectromagnetic hazard mechanism were ever to be discovered in the future, it is very likely that this principle of accepting the risk provided it could be demonstrated to be at an insignificantly low level would come to be applied in due course.

\section{REFERENCES}

[1] British Standards Institution, "British Standard Guide to Prevention of Inadvertent Ignition of Flammable Atmospheres by Radio-Frequency Radiation,' British Standard BS6656, 1986, updated 1991 and 1996. [2] H. Freytag, "Handbuch der Raumexplosionen," Part IIIC. Weinheim, Germany: Verlag Chemie, 1965.

[3] D.P. Howson and G.H. Butcher, "Coaxial breakflash for RF ignition of flammable vapours," Proc. IEE, vol. 122, no. 12, pp. 1453-1454, 1975.

[4] G. Bittner, G. "Über die Funkenzündung explosibler Atmosphäre im Frequenzbereich $1 \mathrm{kHz}$ bis $10 \mathrm{MHz}$," PTB-Mitteilungen, vol. 86, no. 1, pp. 26-30, 1976.

[5] D.P. Howson, P.S. Excell, and G.H. Butcher, "Ignition of flammable gas mixtures by sparks from 2-MHz and 9-MHz sources," Radio and Electronic Engineer, vol. 51, no. 4, pp. 170-174, 1981.

[6] P.S. Excell, "Radio frequency ignition hazards," Hazard Prevention, vol. 20, no. 5, pp. 4-8, 11 and 30, 1984.

[7] P.S. Excell and A.J. Maddocks, "Assessment of worst-case receiving antenna characteristics of metallic industrial structures. Part 1: Electrically-small structures," J. IERE, vol. 56, no. 1, pp. 27-32, 1986.

[8] P.S. Excell and D.P. Howson, "Assessment of worst-case receiving antenna characteristics of metallic industrial structures. Part 2: Electrically-large structures," J. IERE, vol. 56, no. 1, pp. 33-36, 1986.

[9] P.S. Excell, "Ignition of flammable gas mixtures by $\mathrm{X}$-band microwave discharges," Proc. IEE, A, vol. 131, no. 2, pp. 103-110, 1984.

[10] S.S.J. Robertson and R.J. Loveland, "Radio frequency ignition hazards: A review," Proc.IEE, A, vol. 128, no. 9, pp. 608614, 1981.

[11] British Standards Institution, "British Standard Guide to Prevention of Inadvertent Initiation of Electro-Explosive Devices by Radio-Frequency Radiation," British Standard BS6657, 1986, updated 1991 and 1996. [12] J.D. Kraus, Antennas, $2^{\text {nd }}$ ed. New York, NY: McGraw-Hill, 1988, Sect. 12-3.

[13] V.C. Marshall, Major Chemical Hazards. Chichester, U.K.: Ellis Horwood, 1987.

[14] A. Ephreth and D.D. Weiner, "EMC Modelling and Analysis - A Probabilistic Approach," USAF Report No. RADC-TR83-102, 1983

[15] R.A. James, P.S. Excell, and A.Z. Keller, "Probabilistic factors in radio frequency ignition and detonation hazards analyses," Reliability Engineering, vol. 17, no. 2, pp. 139-153, 1987.

[16] P.S. Excell, "A priori estimation of probabilistic factors in EMC: Some experience from RF hazards analysis," in IEE Colloq. Dig. 1992/113 "The Role of Statistics in EMC Specification, Design and Clearance." London, U.K., 1992, pp. 2/1-2/4.

[17] P. Bernardi, S. Pisa, M. Cavagnaro, and E. Piuzzi, IEEE Trans. Antennas \& Propagation, 2001. 